\title{
Conocimiento sobre la proporcionalidad exhibida por futuros profesores en un curso de formación ${ }^{1}$
}

\author{
Knowledge of proportionality exhibited by \\ future teachers in a training course
}

\section{Conhecimento da proporcionalidade exibido por futuros professores em um curso de formação}

Recibido: mayo de 2013

Aceptado: agosto de 2013
Mauro A. Rivas ${ }^{2}$

Walter F. Castro ${ }^{3}$

Patricia M. Konic ${ }^{4}$

\section{Resumen}

En este documento informamos sobre un estudio realizado con una muestra de futuros profesores de primaria, luego de haber concluido su primer cuatrimestre de formación profesional. El estudio estuvo dirigido a determinar el conocimiento sobre la proporcionalidad adquirido por esa muestra, en relación con la distinción entre situaciones proporcionales/no-proporcionales y los tipos de resolución empleados. Los resultados indican, luego de concluido el proceso de instrucción respectivo, la falta de un desarrollo adecuado del razonamiento proporcional para realizar las acciones demandadas.

Palabras clave: Profesor; Formación inicial de profesores; matemáticas escolares; números; relaciones numéricas; razón y proporcionalidad; razonamiento proporcional.

\section{Abstract}

In this paper we report on a study with a sample of future teachers of primary, after having completed its first four months of training. The study was aimed at determining the proportionality knowledge acquired by that sample, in relation to the distinction between situations proportional / non-proportional and employees resolution types. The results indicate, after completion of the respective instructional process, the lack of adequate development of proportional reasoning to perform the actions demanded.

Keywords: Teacher, initial teacher training, school mathematics, numbers, number relationships, reason and proportionality proportional reasoning.

1 Artículo de Investigación.

2 Universidad de Los Andes. Caracas, Venezuela. Contacto: rmauro@ula.ve.

3 Universidad de Antioquia. Medellin, Colombia. Contacto: wfcastro82@gmail.com

4 Universidad de Río Cuarto, Argentina. Contacto: pkonic@unrc.edu.ar 


\section{Resumo}

Neste artigo, relatamos um estudo com uma amostra de futuros professores de primário, depois de ter completado seus primeiros quatro meses de treinamento. O estudo teve como objetivo determinar o conhecimento adquirido pela proporcionalidade que a amostra, em relação à distinção entre situações proporcionais / não-proporcional e tipos de resolução de funcionários. Os resultados indicam, após a conclusão do respectivo processo de instrução, a falta de adequado desenvolvimento do raciocínio proporcional para executar as ações exigidas.

Palavras-chave: Professor, a formação inicial de professores, a matemática da escola, números, relações numéricas, razão e raciocínio proporcional proporcionalidade.

\section{Problemática y marco teórico}

Diversas investigaciones han abordado el conocimiento de la proporcionalidad en la formación de futuros profesores (Ben-Chaim, Keret \& Ilany, 2012; Monteiro, 2003). Los resultados de estos estudios coinciden al señalar que el manejo adecuado del conocimiento sobre esa noción matemática, por parte de los futuros profesores, sigue siendo un problema sin resolver.

Asimismo, la complejidad involucrada en la enseñanza-aprendizaje de la proporcionalidad ha sido objeto de diversos estudios (Ben-Chaim, Keret \& Ilany, 2012; Lamon, 2007). Los estudios realizados parecen identificar, como parte del problema, la falta de desarrollo del razonamiento proporcional o de una idea de razón (Lamon, 2007), lo cual debería ser fomentado desde los niveles de educación básica. En este orden de ideas, la formación del profesir en este tema es trascendental De esto, la necesidad de observar el funcionamiento de los procesos de formación de futuros profesores. Particularmente es necesario indagar si dichos procesos desarrollan conocimiento adecuado que permita distinguir entre situaciones proporcionales/ no-proporcionales y resolver problemas de valor faltante proporcionales (De Bock, Van Dooren, Janssens \& Verschaffel, 2008; Lamon, 2007).

En un trabajo anterior presentamos los resultados sobre las estrategias utilizadas por un grupo de estudiantes de magisterio, en una prueba diagnóstico, al inicio de su formación profesional, al resolver un problema de valor faltante proporcional (Rivas \& Castro, 2011), observándose el predominio del uso de reglas que dan lugar a respuestas correctas sin que un razonamiento proporcional haya tenido lugar (Lamon, 2007). Asimismo, se ha observado en la literatura especializada que los futuros profesores tienen dificultad para distinguir entre situaciones proporcionales y no proporcionales (Ben-Chaim, Keret \& Ilany, 2012).

Ante estos acontecimientos nos preguntamos ¿Están los cursos de formación de futuros profesores atendiendo estos problemas? En este sentido, el propósito de este trabajo es presentar una descripción del conocimiento sobre la proporcionalidad de una muestra de futuros profesores, luego de concluido su primer cuatrimestre de formación profesional. Esta descripción la realizamos con base en las respuestas dadas por la muestra a un ítem de una prueba de control, regularmente aplicada en el desarrollo del curso considerado.

El estudio realizado, cuyo objeto ha sido el conocimiento de la proporcionalidad o el desarrollo del razonamiento proporcional, ha tenido como marco herramientas provenientes del enfoque ontosemiótico (EOS) (Godino, Batanero \& Font, 2007). Específicamente, herramientas de análisis epistémico y cognitivo para el estudio de los problemas considerados y las resoluciones puestas en juego por los sujetos de la muestra ${ }^{5}$. Por razones

5 Ejemplos del uso de este tipo de herramienta puede verse en Rivas y Castro (2011). 
de espacio, en este informe nos hemos limitado a dar cuenta sobre los resultados de las configuraciones cognitivas puestas de manifiesto en las resoluciones.

\section{Metodología}

Este documento forma parte de un proyecto de investigación en proceso en el que se trata de estudiar y fomentar el desarrollo del conocimiento de la proporcionalidad en una muestra de futuros profesores. Sobre la base de la realización de un diagnóstico inicial y el desarrollo de un proceso de instrucción descrito en la Figura 1, se procedió a valorar los conocimientos adquiridos por la muestra por medio de una prueba de control.

Figura. 1: Proceso instruccional llevado a efecto.

\footnotetext{
El proceso de instrucción desarrollado comprende:

1. El desarrollo de una sesión de clase, en la que se incluye la aplicación de la prueba diagnóstico y la puesta en juego una trayectoria didáctica que involucra:

- Presentación de las consignas.

- Exploración personal

- Trabajo cooperativo en equipos para elaborar una respuesta compartida.

- Presentación y discusión

- Institucionalización por el formador, explicitando los conocimientos pretendidos

2. Lectura de materiales sugeridos:

- Fernández, F. (2001). Proporcionalidad entre magnitudes. En E. Castro (Ed.), Didáctica de la matemática en la educación primaria (pp. 533-558). Madrid: Síntesis.

- Godino, J.D., Batanero, C. \& Font, V. (2004) Fundamentos de la enseñanza y aprendizaje de las matemáticas. En J.D. Godino (Dir.), Didáctica de la Matemática para maestros (pp. 5-123). Granada Universidad de Granada. Disponible en: http:// www.ugr.es/local/jgodino. Específicamente lo con cerniente a: Dificultades errores y obstáculos (pp. 73-76).

- Godino, J.D. \& Batanero, C. (2004). Proporcionalidad. En J.D. Godino (Dir.), Didáctica de la Matemática para maestros (pp 271-286). Granada: Universidad de Granada. Disponible en: http://www.ugr.es/local/jgodino.

3. Tutoría sobre las lecturas sugeridas por el for mador atención por medio de la web.

4. Aplicación de la prueba de control.

Fuente: Elaboración propia
}

El estudio del cual ahora informamos comprendió los siguientes momentos: (a) elaboración y análisis epistémico de los ítems de la prueba de control, (b) aplicación de la prueba que incluye los ítems correspondientes y (c) análisis cognitivo de las respuestas dadas por los sujetos de la muestra a los ítems respectivos. Por razones de espacio, en este documento, referiremos sólo a este último momento.

La muestra es de tipo incidental (León \& Montero, 2003), en la que el grupo de sujetos está previamente constituido por la condición de estar iniciando la carrera de magisterio en el curso y sección seleccionado. La muestra se encuentra conformada por 59 sujetos.

El instrumento aplicado es un ítem (número 6) de una prueba de control, aplicada al final del primer cuatrimestre, del primer año de la carrera de magisterio. Una transcripción de ese ítem se presenta en la Figura 2.

El ítem (número 6) de la prueba de control está dirigido a evaluar básicamente dos aspectos relacionados con el desarrollo del razonamiento proporcional, a saber: (a) distinguir entre situaciones proporcionales y no proporcionales, y (b) resolver situaciones de proporcionalidad del tipo valor faltante. En este sentido las situaciones propuestas son: en el problema (a) una situación proporcional de valor faltante y en los problemas (b) y (c) dos situaciones pseudo-proporcionales (ver Figura 2).

Figura 2: Ítem de la prueba de control aplicada

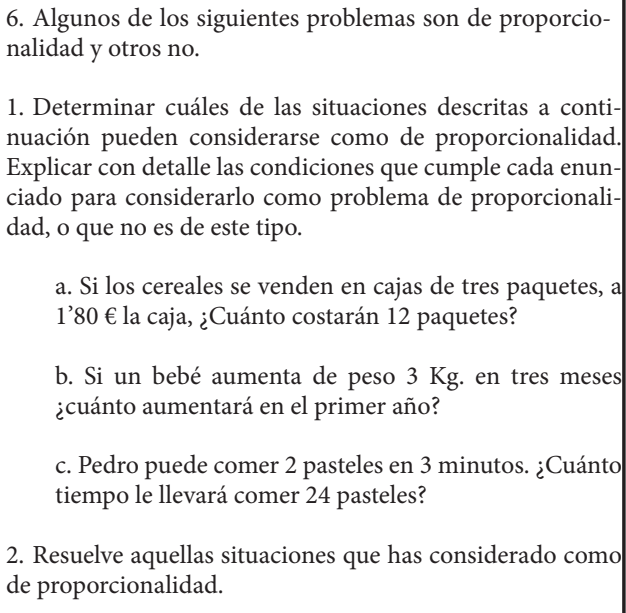

6. Algunos de los siguientes problemas son de proporcionalidad y otros no.

1. Determinar cuáles de las situaciones descritas a continuación pueden considerarse como de proporcionalidad. Explicar con detalle las condiciones que cumple cada enunciado para considerarlo como problema de proporcionalidad, o que no es de este tipo.

a. Si los cereales se venden en cajas de tres paquetes, a 1 '80 € la caja, ¿Cuánto costarán 12 paquetes?

b. Si un bebé aumenta de peso $3 \mathrm{Kg}$. en tres meses ¿cuánto aumentará en el primer año?

c. Pedro puede comer 2 pasteles en 3 minutos. ¿Cuánto tiempo le llevará comer 24 pasteles?

2. Resuelve aquellas situaciones que has considerado como de proporcionalidad. 


\section{Análisis de datos}

Una vez aplicada la prueba de control se procedió a hacer el análisis de las respuestas dadas por los sujetos. Nos limitaremos a presentar los resúmenes de las respuestas en relación con la distinción entre proporcionalidad/no-proporcionalidad de las situaciones y los tipos de resolución puestos en juego por los sujetos.

En la Tabla 1 presentamos los resultados de los juicios a las situaciones planteadas en los tres problemas del instrumento, en correspondencia con la respuesta a la parte 6.1 del ítem en cuestión (Figura 2).

Tabla 1: Reconocimiento de la proporcionalidad o no-proporcionalidad $(\mathrm{N}=59)$

\begin{tabular}{|l|r|r|r|}
\hline Tipo de respuesta & Problema (a) & Problema (b) & Problema (c) \\
\hline Si es proporcional & $51(86,4 \%)$ & $26(44,1 \%)$ & $51(86,4 \%)$ \\
\hline No es proporcional & $7(11,9 \%)$ & $31(52,5 \%)$ & $5(8,5 \%)$ \\
\hline Condicional & - & $2(3,4 \%)$ & $3(5,1 \%)$ \\
\hline No responde & $1(1,7 \%)$ & - & \\
\hline
\end{tabular}

Fuente: Elaboración propia

Se observa en la Tabla 1, que siendo el problema (a) un problema de valor faltante proporcional, relación precio/paquetes o cajas, 7 sujetos $(11,9 \%)$ consideran que el problema no es de proporcionalidad. En el problema (b), de tipo pseudo-proporcional, relación peso/edad de un bebe, 26 sujetos (44.1\%) consideran que el problema es de proporcionalidad. Mientras en el problema (c), de tipo pseudo-proporcional, relación pasteles/minuto, 51 sujetos $(86,4 \%)$ consideran que el problema es de proporcionalidad.

En la Tabla 2 presentamos los resultados de los tipos de resolución puestos en juego por los sujetos de la muestra. Estas respuestas corresponden con la parte 6.2 del ítem en cuestión (Figura 2).

Se debe señalar que aún cuando sólo el problema (a) plantea una situación proporcional del tipo valor faltante (los problemas (b) y (c) no son de proporcionalidad) el error cometido por los sujetos al juzgar de manera incorrecta las situaciones pseudo-proporcionales como proporcionales los condujo a proveer de una resolución para esos problemas.

Se observa en el problema (a) (ver Tabla 2) una mayor tendencia del uso de la regla de tres como procedimiento de resolución (22 sujetos, 37,3\%). Esta tendencia tiene mayor preponderancia en las resoluciones dadas a los problemas (b) y (c).

Tabla 2: Tipos de resolución

\begin{tabular}{|l|r|r|r|}
\hline Tipo de resolución & Problema (a) & Problema (b) & Problema (c) \\
\hline Regla de tres (R-3) & $22(37,3 \%)$ & $21(35,6 \%)$ & $35(59,3 \%)$ \\
\hline $\begin{array}{l}\text { R-3 y otro } \\
\text { procedimiento }\end{array}$ & $7(11,9 \%)$ & $2(3,4 \%)$ & $5(8,5 \%)$ \\
\hline $\begin{array}{l}\text { Otro procedimiento } \\
\text { No proporcional }\end{array}$ & $22(37,3 \%)$ & $5(8,5 \%)$ & $14(23,7 \%)$ \\
$\begin{array}{l}\text { No resuelve / No } \\
\text { responde }\end{array}$ & $7(11,9 \%)$ & $31(52,5 \%)$ & $5(8,5 \%)$ \\
\hline
\end{tabular}

Fuente: Elaboración propia

\section{Conclusiones}

Los resultados presentados en la Tabla lindican que los futuros profesores han exhibido dificultad para distinguir entre situaciones proporcionales y no proporcionales.

Los resultados presentados en la Tabla 2 muestran que los futuros profesores manifiestan preferencia por el uso de procedimientos basados en reglas para resolver problemas proporcionales. El uso de este tipo de procedimientos puede dar lugar a una solución correcta sin que tenga lugar el razonamiento proporcional que corresponde.

Ambos resultados parecen indicar la falta de un desarrollo adecuado del razonamiento proporcional, que permita distinguir entre situaciones proporcionales/no-proporcionales y la puesta en juego de procedimientos de resolución que muestren evidencias de ese desarrollo. Finalmente, estas manifestaciones indican que ese desarrollo no ha sido logrado por medio del proceso de instrucción llevado a efecto. 


\section{Referencias}

Ben-Chaim, D., Keret, Y. \& Ilany, B. (2012). Ratio and proportion. Research and teaching in mathematics teachers' education. Rotterdam: Sense Publishers.

De Bock, D., Van Dooren, W., Janssens, D. \& Verschaffel, L. (2008). The illusion of linearity: From analysis to improvement. New York: Springer Science+Business Media, LLC.

Godino, J.D., Batanero, C. \& Font, V. (2007). The onto-semiotic approach to research in mathematics education. ZDM The International Journal on Mathematics Education, 39 (1-2), 127-135.

Lamon, S. J. (2007). Rational numbers and proportional reasoning: Toward a theoretical framework for research. En F. K. Lester (Ed.), Second handbook of research on mathematics teaching and learning (Vol. 1, pp. 629-667). Charlotte, NC: Information Age Publishing.

León, O. \& Montero, I. (2003). Diseño de Investigaciones. Madrid: McGraw-Hill.

Monterio, C. (2003). Prospective elementary teachers' misunderstanding in solving ratio and proportion problems. En N. Pateman, B. Dougherty \& J. Zilliox (Eds.), Proceedings of the 27th Conference of the International Group for the Psychology of Mathematics Education (Vol. 3, pp 317-323). Honolulu, HI: PME.

Rivas, M.A. \& Castro, W.F. (2011). Aportes del estudio de las configuraciones epistémicas y cognitivas sobre la proporcionalidad en la formación inicial de profesores de primaria. En G. García Oliveros (Comp.), Memorias del 12 ECME, Quindío, Colombia. 